\title{
Predictors of high-quality cord blood units
}

\author{
Sandra V.F. Santos, ${ }^{1}$ Sonia M.O. Barros, ${ }^{1}$ Marisa S. Santos, ${ }^{2}$ Luciana C. Marti, ${ }^{3}$ \\ Andreza A.F. Ribeiro, ${ }^{4}$ Andrea T. Kondo, ${ }^{4}$ and Jose M. Kutner ${ }^{4}$
}

BACKGROUND: Analysis of umbilical cord blood (UCB) transplants shows a correlation between engraftment and total number of infused cells. Thus, it is worth evaluating what maternal and neonatal characteristics and collection techniques may affect the quality of UCB units.

STUDY DESIGN AND METHODS: A cross-sectional study was performed with 7897 donors sequentially selected in three health care institutions in Brazil from October 2004 to March 2012, in which both quantitative and qualitative approaches were applied. All donors were considered suitable for cord blood collection.

RESULTS: The maternal and neonatal characteristics and techniques of collection that influenced the total number of nucleated cells (TNCs; $p<0.001$ ) were type of delivery, newborn weight and sex, and institution of UCB collection. The TNC count was associated with gestational age $(p=0.008)$, type of delivery $(p<0.001)$, newborn sex $(p<0.001)$, newborn weight $(p<0.001)$, and UCB collection technique $(p=0.003)$. Center B presented the largest number of nucleated cells in its results $(p<0.001)$, followed by Center $A(p=0.001)$. Other characteristics, such as maternal age, were analyzed but were not relevant for the nucleated cell number.

CONCLUSION: This study provides elements for a model that allows an efficient selection of UCB donors, prioritizing candidates who have a better chance to lead to an optimized use of cord blood cells units.
$\mathrm{U}$ mbilical cord blood (UCB) is a rich source for hematopoietic stem cells, which have been used for the treatment of various hematologic disorders in children and adults. Hematopoietic stem cells are found in the marrow, in the UCB, and after stimulus in peripheral blood. These cells are crucial for therapeutic procedures of long-term hematopoiesis reconstitution, even after myeloablative conditioning.

Using UCB for allogeneic transplants has made possible increasing numbers of these procedures, due to the feasibility of performing transplants with mismatched HLA. This property has benefited the Brazilian population, which is highly multiracial. It has also benefited patients carrying rare HLA haplotypes.

However, the volume and number of stem cells are usually low in UCB units, posing important limitations for transplantation. Thus, the use of a UCB unit that has adequate volume, cellularity, and viability is very important for the success of the transplant. A constant investigation of the best criteria for achieving high-quality UCB units is necessary.

ABBREVIATIONS: C-section $=$ cesarean section; TNCs $=$ total number of nucleated cells; $\mathrm{UCB}=$ umbilical cord blood.

From the ${ }^{1}$ Universidade Federal de São Paulo-UNIFESP, Escola Paulista de Enfermagem, São Paulo, Brazil; the ${ }^{2}$ Instituto Nacional de Cardiologia, Rio de Janeiro, Brazil; and the ${ }^{3}$ Instituto Israelita de Ensino e Pesquisa Albert Einstein and the ${ }^{4}$ Departamento de Hemoterapia, Hospital Israelita Albert Einstein, São Paulo, Brazil.

Address reprint requests to: Sandra Valeria Francisconi Santos, Street Hilário de Gouveia, 126 Apartment 302, Copacabana, Rio de Janeiro, Brazil, 22040020; e-mail: sandra.vale21@ hotmail.com.

Received for publication December 22, 2015; revision received April 4, 2016; and accepted April 7, 2016.

doi:10.1111/trf.13653

(C) $2016 \mathrm{AABB}$

TRANSFUSION 2016;56;2030-2036 
Good results for transplantation of cord blood units are difficult to obtain, but some associated factors are predictable, such as the unit quality. One of the main criteria for considering a UCB unit for transplantation is the total number of nucleated cells (TNCs) collected. The minimum required TNCs in UCB for processing has increased over the years. Initially this number was between $5 \times 10^{8}$ and $8.5 \times 10^{8}$. Arrojo and colleagues ${ }^{1}$ suggest the need for a higher TNCs $\left(10 \times 10^{8}\right)$, indicating that TNC count is an important factor affecting quality of UCB and placental units.

The optimal amount of volume and cells (dose) in a UCB unit has not been clearly defined, although usually high volume units are related to high cellularity. Previous studies strongly correlate hematopoietic recovery and graft rejection with TNCs and the recipient weight, ${ }^{2,3}$ which leads the UCB and placental banks to give preference to providing units for children and adults of low body weight. The minimum number of cells required for a unit to be transplanted is not a consensus; it depends on factors such as HLA compatibility and the underlying disease being treated. Suggested variables have been at least $3 \times 10^{7} \mathrm{TNCs} / \mathrm{kg}$ or at least $1.5 \times 10^{5} \mathrm{CD} 34+/ \mathrm{kg}$ for a successful transplantation. ${ }^{4,5}$ Some studies have shown that the quality of UCB units can be influenced by several obstetric and neonatal factors and by the collection technique. ${ }^{6,7}$

Thus, our study aimed to investigate the factors involved in UCB collection to obtain a better use and quality of the harvested material and increase the likelihood of transplantation. Our results may improve our current understanding ${ }^{8,9}$ of the main factors affecting the quality of UCB units.

To this end, we evaluated maternal and gestational age; type of delivery and pregnancy; neonatal weight, sex, and race; and whether cell collection was intrauterine, extrauterine, or intra- and extrauterine, in three different health care institutions. The primary outcome used was the total number of cells $\left(\geq 12.5 \times 10^{8}\right)$ and the cell volume of the UCB unit.

\section{MATERIALS AND METHODS}

This study was evaluated and approved by the Ethical Committee of the Albert Einstein Hospital in São Paulo, Brazil, under CAAE number 02623012.7.0000.5505. This is a cross-sectional, retrospective study in which both quantitative and qualitative approaches were applied. A survey was conducted in the Public Umbilical Cord Blood Bank (Department of Hematology) of the Albert Einstein Hospital in São Paulo, Brazil. This blood bank receives cord blood units from three institutions, here named as Center A, Center B, and Center C. The stem cell laboratory is located in Center A so it is where the units were evaluated for further processing, freezing, storage, and release for transplantation of hematopoietic stem cells. We studied 8513 cord blood records from the public UCB bank. The data were collected for 8 years (from October 4, 2004, until March 31, 2012). A total of $96 \%$ of these records (7897) that met the inclusion criteria were included in this study.

\section{Inclusion criteria}

UCB reception forms, processing, and freezing samples containing complete records regarding newborn weight and race, maternal age, gestational age, type of delivery (vaginal or cesarean), type of pregnancy (single or double), cord blood collection technique (intrauterine, extrauterine, or combination of intra- and extrauterine), and collecting institution were considered.

\section{Exclusion criteria}

Reception forms containing incomplete or inadequate records regarding the variables selected for the study were excluded.

\section{Independent variables}

Newborn characteristics

Newborn characteristics included were weight (g), sex (male or female), and race/color (defined as the feature stated on the certificate of live birth): white and nonwhite (black, Asian, and mixed race).

\section{Maternal characteristics}

Maternal characteristics included age (years), gestational age (defined as the time measured in weeks and full days elapsed since the last menstrual period), delivery type (categorized into cesarean or vaginal), and pregnancy (categorized as single or twin).

\section{Technique of collection}

UCB collection techniques were categorized into 1) intrauterine, 2) extrauterine, or 3) intra- and extrauterine.

1. Intrauterine collection (before handling the placenta): This technique is used to collect the cord blood just after birth when the newborn is under the care of the neonatologist. After umbilical cord section, the professional responsible for the collection performs antisepsis of the vein to prevent contamination by microorganisms or maternal blood. The umbilical vein is punctured in the distal third of the umbilical cord with a needle coupled to a bag (a closed system containing anticoagulant) and the drainage of the whole blood is performed into the collection bag by gravity.

2. Extrauterine collection (after handling the placenta): A health care professional performs antisepsis of the umbilical cord and placenta vessels. Next, several punctures in the cord and placenta veins are made, gradually transferring the collected blood to the 
collection bag device. At the end of extrauterine collection, the health care professional cleans and seals the collection bag.

3. Intra- and extrauterine collection technique: This is done whenever the two techniques can be combined to obtain a higher cell volume. The final volume is the sum of the volume obtained with each technique, which is then recorded on the collection form.

\section{Institution of UCB collection}

Three institutions named Center A, Center B, and Center $\mathrm{C}$ were included in this study. The UCB collection in the three centers followed the same operational procedure.

\section{Dependent variable}

The TNCs before being processed correspond to the number of white blood cells and red blood cells $\times 10^{6} / \mathrm{L}$ UCB times the total volume of umbilical cord and placental blood collected without anticoagulant.

\section{Statistical analysis}

To evaluate the relationship between the explanatory variables and TNC count of more than $12.5 \times 10^{8}$ we used a logistic regression model in multiple approaches. These analyses were performed with computer software (SPSS Statistics for Windows, Version 17.0, released 2008, SPSS, Inc.).

\section{RESULTS}

\section{Descriptive analysis}

The database contains records from 7897 UCB collections, of which 5157 (65.3\%) samples were frozen and 2740 $(34.7 \%)$ were discarded due to low volume and/or low TNC count. Table 1 shows the descriptive analysis of the variables observed in all samples.

\section{Inferential analysis}

For a better fit of the multiple regression models, the variable "twin/single pregnancy" was not included due to a strong association with newborn weight and gestational age. ${ }^{10}$ Thus, for a multiple logistic regression model we considered the following variables: maternal age, gestational age, delivery type, sex, race, newborn weight, type of collection, and collecting institution. An association was investigated between these variables and the UCB quality, as defined by TNC count of more than $12.5 \times 10^{8}$. The results of this model are shown in Table 2; the model adjustment was verified by a residual plot (Fig. 1). The deviation component chart by percentile of the standard normal distribution shows adequacy of the model built for the outcome TNC count of more than $12.5 \times 10^{8}$, since

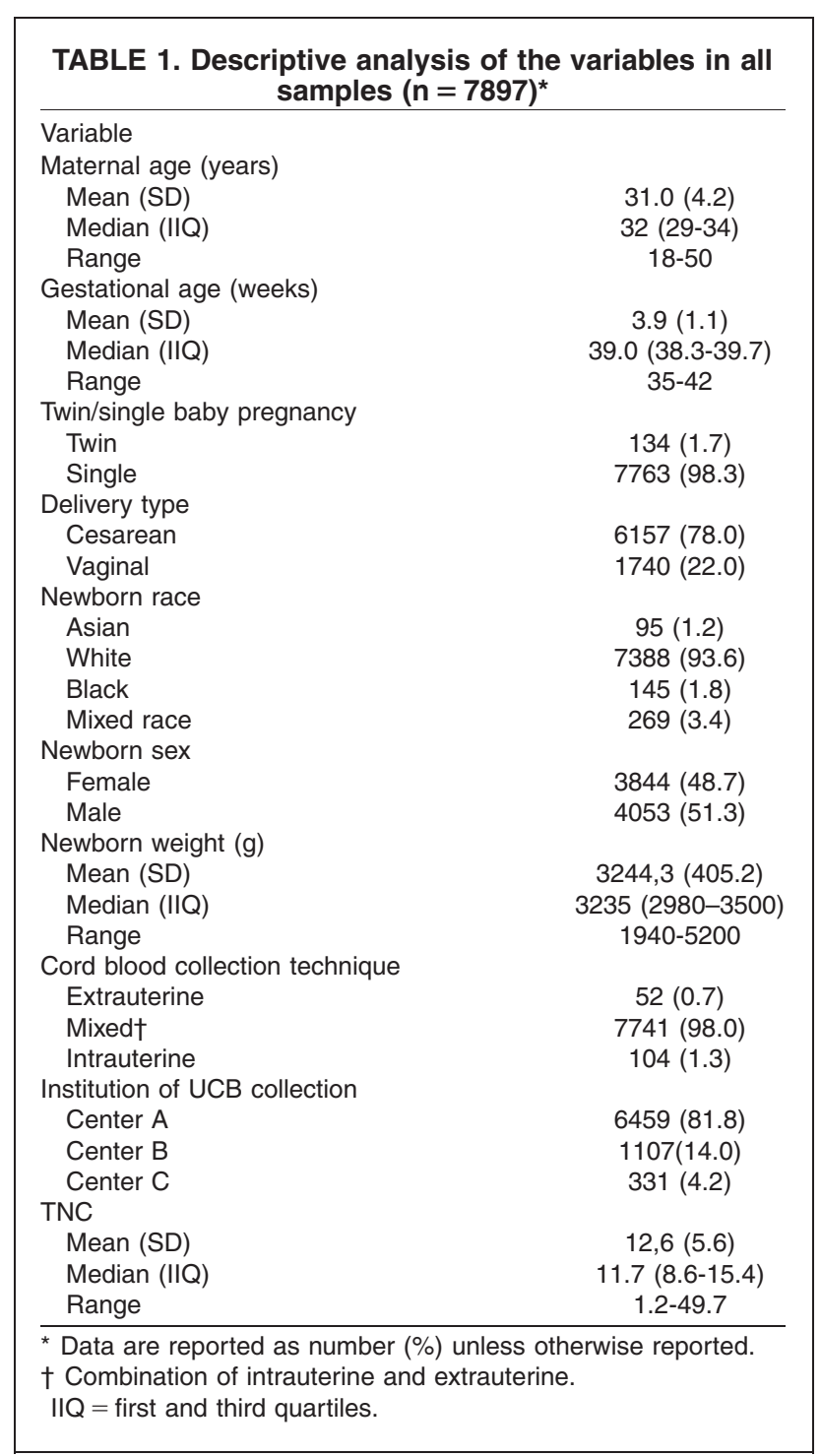

the points are close enough to the reference curve and within the $95 \%$ confidence range.

There was no association between maternal age and product quality. Regarding gestational age, we observed that collections performed between 39 and 40 weeks of pregnancy led to products with $46 \%$ higher chances of better quality than collections performed during Weeks 35 and 36. We also found that $43 \%$ more UCB collections were from cesarean sections (C-sections) than from vaginal deliveries.

Concerning newborn weight, we observed that UCB collections of higher-weight newborns are more likely to present a quality product (odds ratio [OR], 2.6 in the category 2501-3000 g; 5.6 in the category 3001-3500 g; 11.7 in category 3501-4000 g; and 24 in category $>4000$ g) compared to lower-weight newborns (category $\leq 2500 \mathrm{~g}$ ).

We also observed that the intra- and extrauterine combined UCB collection technique showed products with 2.6 times better chances of quality than UCB 
TABLE 2. Association between product quality defined by TNC count of more than $12.5 \times 10^{8}$ and all variables of interest in all samples

\begin{tabular}{|c|c|c|c|}
\hline Variables & Categories & OR (95\% Cl) & $p$ value \\
\hline \multirow[t]{5}{*}{ Newborn weight } & $\leq 2500$ & 1.00 & \\
\hline & $2501-3000$ & $2.62(1.75-3.94)$ & $<0.001$ \\
\hline & $3001-3500$ & $5.64(3.77-8.45)$ & $<0.001$ \\
\hline & $3501-4000$ & $11.71(7.75-17.70)$ & $<0.001$ \\
\hline & $>4000$ & 24.00 (14.66-39.29) & $<0.001$ \\
\hline \multirow[t]{2}{*}{ Newborn sex } & Female & $1.57(1.43-1.73)$ & $<0.001$ \\
\hline & Male & 1.00 & \\
\hline \multirow{2}{*}{ Newborn race } & White & $1.23(0.99-1.53)$ & 0.067 \\
\hline & Not white & 1.00 & \\
\hline \multirow[t]{4}{*}{ Gestational age } & $35-36$ & 1.00 & \\
\hline & $37-38$ & $1.30(0.99-1.71)$ & 0.062 \\
\hline & $39-40$ & $1.46(1.10-1.93)$ & 0.008 \\
\hline & $>40$ & $1.41(0.98-2.05)$ & 0.068 \\
\hline \multirow[t]{2}{*}{ Delivery type } & Cesarean & $1.43(1.26-1.62)$ & $<0.001$ \\
\hline & Vaginal & 1.00 & \\
\hline \multirow[t]{2}{*}{ Cord blood collection technique } & Extrauterine or intrauterine & 1.00 & \\
\hline & Extrauterine and intrauterine & $2.62(1.76-3.90)$ & 0.003 \\
\hline \multirow[t]{3}{*}{ Institution of UCB collection } & Center C & 1.00 & \\
\hline & Center A & $1.51(1.17-1.94)$ & 0.001 \\
\hline & Center B & $1.59(1.20-2.10)$ & $<0.001$ \\
\hline
\end{tabular}

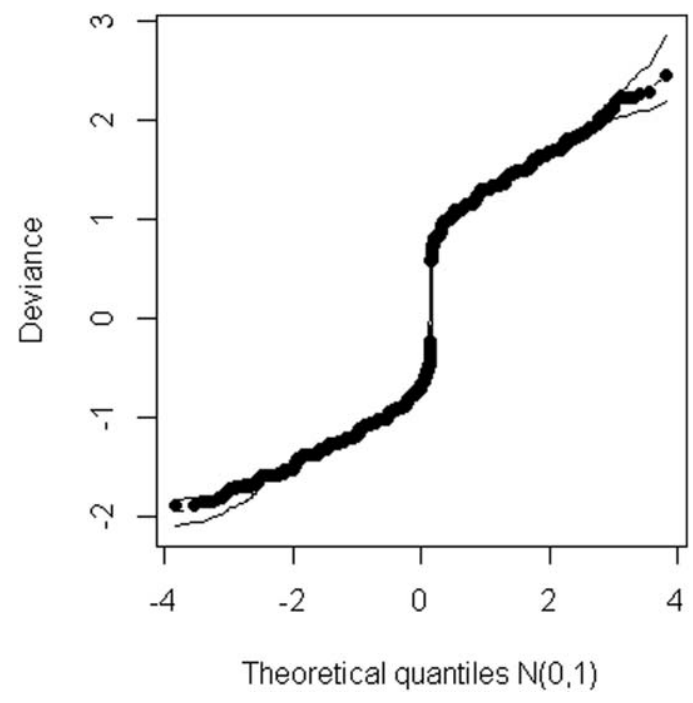

Fig. 1. Residual plot. This plot contains the ordered deviance residuals of the multivariate logistic regression model fitted for our data versus the standard normal quantiles, with simulated confidence bands. We did not identify observations with deviance residual outside the bands, which means that we had an indication of goodness of the final model fit. This analysis follows the recommendations of Pregibon. ${ }^{11}$

collections using only intra- or only extrauterine technique. Center A and Center B showed higher chances of adequate units (51 and 59\%, respectively) than Center C.

\section{DISCUSSION}

Several analyses were carried out to determine the main dependent variable associated with high-quality UCB units, which are represented by higher TNC cells in the unit. All variables analyzed are discussed individually as follows.

\section{Maternal age}

There is an increasing number of pregnant women at an advanced age (older than 37 years) ${ }^{12,13}$ and UCB might be collected from these women as well, hence the need to investigate whether advanced age affects the quality or number of collected cells. ${ }^{12,13}$ Lampinen and colleagues, ${ }^{14}$ in a review about maternal age in women over 35 years, concluded that we need to carry out observational studies involving pregnant women with advanced age, to increase understanding and knowledge in relation to maternal age and risks to pregnant women and their fetuses. Thus, in this study we investigated whether the older age groups would be associated with higher rates of low-quality UCB units. However, multiple logistic regression analysis found no significant association between maternal age and the TNCs, indicating that maternal age is not a variable affecting UCB quality, and thus pregnant women with advanced age are also suitable as UCB donors.

\section{Gestational age}

Since 2004, Brazilian legislation has called for the public cord blood banks to conduct their collections in pregnant women with gestational age equal to or over 35 weeks. This is justified by the increased TNCs that occurs between 32 and 41 weeks' gestational age, probably due to greater development of the fetus. ${ }^{15}$

We noted that the greater the gestational age the higher the TNCs, a finding that agrees with the results of Keersmaekers and coworkers. ${ }^{16}$ In addition, Kurtzberg and 
coworkers $^{2}$ find that larger term babies of any ethnicity are more likely to produce cord blood units with higher volumes and nucleated and CD34+ cell contents. This may be a critical factor for donor selection, since the chance of successful quality units between 39 and 40 weeks is $46 \%$ higher.

\section{Delivery type}

There are several hypotheses to explain the better UCB quality results obtained with C-section delivery compared to vaginal birth. One of them is that UCB collection is facilitated during cesarean delivery, since elective C-sections are always scheduled. Also, higher TNCs in C-sections may be explained by the faster UCB clamping, which favors the maintenance of blood flow, allowing collection of greater volume of blood, as described by Rodrigues and colleagues. ${ }^{17}$ Higher TNC counts in Csections is also shown in a study by Kurtzberg and colleagues. $^{2}$

Another important determinant of UCB quality is physiologic placenta delivery, which is more frequent in vaginal than cesarean deliveries. During the C-section, manual handling of the placenta is recurrent and reduces the possibility of blood clots in the collected UCB. This hypothesis was suggested by Aufderhaar and coworkers ${ }^{18}$ who found that manual placenta removal increases the viable cell numbers and leads to higher-quality UCB storage units.

Although several studies show higher cell numbers in UCB units obtained in C-sections than in vaginal delivery, we cannot forget that vaginal delivery is more beneficial to the mother and newborn. ${ }^{19}$ Thus, optimizing UCB collection will include units from vaginal delivery.

\section{Newborn sex}

No difference between sex of the infant donors of the UCB unit has been associated with the quality of the unit. However, when we analyzed the results for the TNCs, we observed significant differences between sexes. TNC was higher in units collected from female newborns $(p<0.001)$ than those from male newborns. The smaller TNC counts in UCB collected from male newborns has been described by Solves and coworkers, ${ }^{20}$ although, from the clinical point of view, this difference is considered small and of uncertain meaning. Our findings were comparable to those reported by Kurtzberg and colleagues who showed equivalent volumes from cord blood units collected from female and male infants, but higher cell counts were obtained from female infants than from male infants. $^{2}$

\section{Newborn race}

The reason to consider race as a variable was suggested in a previous work, ${ }^{21}$ which evaluated several institutions with varied racial composition of newborns. In addition, it is necessary to improve the racial diversity currently found in Brazilian blood banks. There is great difficulty in finding matching donors, while in other countries the chances are much higher (30 times higher). According to a study conducted by the National Register of Bone Marrow Donors (REDOME), the difficulty in finding a donor results from the small representation of racial minorities in cord blood banks and the low proportion of donation rates among individuals of these groups within the demographic areas.

Another problem, besides the low rate of finding matching donors, relates to the high costs in performing international bank searches. According to Samuel and coworkers, $^{22}$ a cord blood unit from an international donor costs between $\$ 20,000$ and $\$ 30,000$ while the cost of the same cord blood unit in the country would be threefold lower. A strategy adopted by the health ministry, through BrasilCord network, was to contemplate the racial diversity of our population by expanding collection to institutions that did not participate in previous collections.

Therefore, in this study race was categorized as white and nonwhite (mixed race, Asian, and black). ${ }^{23}$ We did not observe any significant association between race and TNC counts when adopting the logistic regression model (Table 2). This result was in contrast to the study by Kurtzberg and coworkers, which found differences in TNC counts between African Americans and all the other ethnicities for the same volume of cells. ${ }^{2}$ The differences observed between these studies are probably due to differences in the analysis applied in each of them. Because we separated the population between white and nonwhite individuals (black, Asian, and mixed race), we may have underestimated the differences between the two groups. In the other study, the population was separated into five groups. Another difference between the studies is the fact that the population in North America has characteristics different from those of the Brazilian population. ${ }^{24}$

\section{Newborn weight}

Previous studies ${ }^{2,25,26}$ highlighted the relationship of newborn weight with the quality of the UCB unit, corroborating our findings of an association between newborn weight and TNCs (Table 2). George and colleagues ${ }^{27}$ consider the ideal newborn weight as more than $3000 \mathrm{~g}$. In this study, we used the cutoff proposed by Bart and coworkers $^{28}\left(12.5 \times 10^{8} \mathrm{TNCs}\right)$ to determine the optimum UCB unit. Our findings show that collections from newborns with heavier weight ( $>3000 \mathrm{~g}$ ) have an OR of 5.6 in the category 3001 to $3500 \mathrm{~g}$ and 24 in the category more than $4000 \mathrm{~g}$. Consequently, newborn weight is an easy and reliable screening criterion for high-quality UCBs. 


\section{Cord blood collection technique}

The blood collection technique is one of the predictors for obtaining a high-quality UCB unit. The intrauterine technique concerns collection while the placenta is still in the uterus; collection is interrupted only when the umbilical vein flow ceases. The good volume obtained by this technique is attributed to the natural blood flow from the umbilical cord and uterine contractions. As for the extrauterine technique, it is performed immediately after handling the placenta. ${ }^{29}$ Coagulation may result in disposal of the UCB unit due to low volume of cells and consequently low TNCs. The mixed UCB collection technique (which combines both intra- and extrauterine techniques) is the best option to obtain nucleated cells (Table 2). Although the UCB volume represents a minor indicator of quality, ${ }^{21}$ the higher the volume the greater the cell number and the chances of UCB use, ensuring greater probability of success in transplantation. ${ }^{21}$ Keersmaekers and coworkers ${ }^{16}$ found that each 10 -mL increase in the unit volume corresponds to more than twice the likelihood of reaching the minimum unit of nucleated cells required for storage.

\section{Institution of UCB collection}

The choice of the institution for UCB collection has to be well planned, and several factors should be considered before an institution is officially designated for UCB collection. For instance, the number of deliveries per month, positive serology rates, and high-risk pregnancy rates are all considered. The knowledge and acceptance of professionals and their adherence to the UCB donation process also need to be verified. It is essential to invest in training and awareness of health professionals involved with the collection process, including nurses, technicians, pediatricians, anesthetists, and obstetricians. ${ }^{30}$

Center A is the collection center in which the operating period was longest (October 2004 to March 2012), which may explain the good results. The first center for external collection, Center C, represented a challenge for our study because it needed several adaptations to take part in our study, including staff training and development of a sense of commitment to the procedure. It is possible that the lack of expertise in external collection impacted the results obtained with this institution. Center $B$ was included in the study after undergoing the same adaptations already done for Center $\mathrm{C}$ in regard to external collection of UCB. The team commitment to standard procedures, the need for training, and constant supervision have been highlighted by Roh and coworkers, ${ }^{30}$ who discussed the role of obstetricians in guiding donors as well in the collection procedures and showed that lack of knowledge about the collection procedure can reduce donor numbers.

\section{Statistics limitations}

A multivariate statistical analysis determined the factors that mostly affect the cord blood unit collected for storage and eventual transplantation. Our analysis has some limitations; for instance, the training of health professionals was not included.

Another statistical limitation is related to the categorized variables. Our findings do not exclude a good unit that does not follow these variables; however, following the variables will highly increase the probability of obtaining better units.

In conclusion, a significant cost is involved in collection and storage of cord blood units, and thus knowing which births may produce good quality UCB could optimize costs and help avoid collection of samples that most likely will not result in good quality UCB. This study provides elements for a model that may optimize selection of cord blood donors, prioritizing candidates whose UCB has high chances of being of good quality. The variables that most positively influenced the cord blood unit quality were gestational age (39-40 weeks), C-section delivery, use of mixed collection technique, and newborn weight of more than $4000 \mathrm{~g}$. While the birth method and collection method should be determined according to the best care for mothers and newborns, the gestational age and newborn weight are variables that have no effect on the mother's or newborn's well-being and represent information that is readily available. Thus, these variables are the most suitable to be used when choosing donors with the best chances of yielding good-quality UCBs.

\section{ACKNOWLEDGMENTS}

We are thankful to the blood bank, cryopreservation laboratory, and special techniques clinical laboratory staff; to the donors, the obstetric center physicians, and their teams; and to the nurses involved in the UCB collection and for all SBIBHAE support.

\section{CONFLICT OF INTEREST}

The authors have disclosed no conflicts of interest.

\section{REFERENCES}

1. Arrojo IP, Lamas Mdel C, Verdugo LP, et al. Trends in cord blood banking. Blood Transfus 2012;10:95-100.

2. Kurtzberg J, Cairo MS, Fraser JK, et al. Results of the cord blood transplantation (COBLT) study unrelated donor banking program. Transfusion 2005;45:842-55.

3. Chao NJ. Selection of an umbilical cord blood graft for hematopoietic cell transplantation [Internet]. Boston: UpToDate; 2014 [cited 2014 Jul 28]. Available from: http:// www.uptodate.com/contents/selection-of-an-umbilicalcord-blood-graft-for-hematopoietic-cell-transplantation? source $=$ see_link\&anchor $=$ H61604479\#. 
4. Barker JN, Scaradavou A, Stevens CE. Combined effect of total nucleated cell dose and HLA match on transplantation outcome in 1061 cord blood recipients with hematologic malignancies. Blood 2010;115:1843-9.

5. Eapen M, Klein JP, Ruggeri A, et al. Impact of allele-level HLA matching on outcomes after myeloablative single unit umbilical cord blood transplantation for hematologic malignancy. Blood 2014;123:133-40.

6. Jones J, Stevens CE, Rubinstein P, et al. Obstetric predictors of placental/umbilical cord blood volume for transplantation. Am J Obstet Gynecol 2003;188:503-9.

7. Page KM, Mendizabal A, Betz-Stablein B, et al. Optimizing donor selection for public cord blood banking: influence of maternal, infant, and collection characteristics on cord blood unit quality. Transfusion 2014;54:340-52.

8. Querol S, Gomez SG, Pagliuca A, et al. Quality rather than quantity: the cord blood bank dilemma. Bone Marrow Transplant 2010;45:970-8.

9. Wen SH, Zhao WL, Lin PY, et al. Associations among birth weight, placental weight, gestational period and product quality indicators of umbilical cord blood units. Transfus Apher Sci 2012;46:39-45.

10. Harris DT, Brown HL, Grossman JD, et al. Retrospective review of umbilical cord blood collections: singleton versus multiple births. Obstet Gynecol 2002;99:85S-6S.

11. Pregibon D. Logistic regression diagnostics. Ann Stat 1981;9: 705-24.

12. Huang L, Sauve R, Birkett N, et al. Maternal age and risk of stillbirth: a systematic review. CMAJ 2008;178:165-72.

13. Martin JA, Hamilton BE, Ventura SJ, et al. Births: final data for 2010. Natl Vital Stat Rep 2012;61:1-72.

14. Lampinen R, Vehvilainen-Julkunen K, Kankkunen P. A review of pregnancy in women over 35 years of age. Open Nurs J 2009;3:33-8.

15. Kogler G, Callejas J, Sorg RV, et al. An eight-fold ex vivo expansion of long-term culture-initiating cells from umbilical cord blood in stirred suspension cultures. Bone Marrow Transplant 1998;21Suppl3:S48-53.

16. Keersmaekers CL, Mason BA, Keersmaekers J, et al. Factors affecting umbilical cord blood stem cell suitability for transplantation in an in utero collection program. Transfusion 2014;54:545-9.

17. Rodrigues CA, Pereira NF, Oliveira DC, et al. Transplante de sangue de cordão umbilical—SCU. Rev Bras Hematol Hemoter 2010;32:8-12.
18. Aufderhaar U, Holzgreve W, Danzer E, et al. The impact of intrapartum factors on umbilical cord blood stem cell banking. J Perinat Med 2003;31:317-22.

19. Resick LK, Erlen JA. Vaginal birth after cesarean: issues and implications. J Am Acad Nurse Pract 1990;2:100-6.

20. Solves P, Perales A, Mirabet V, et al. Optimizing donor selection in a cord blood bank. Eur J Haematol 2004;72: 107-12.

21. Valeria SS, Marti L, Ribeiro AA, et al. A cross-sectional study of umbilical cord blood donor profiles and their influence on umbilical cord blood collection in a Brazilian hospital. Cytotherapy 2011;13:1120-7.

22. Samuel GN, Kerridge IH, Vowels M, et al. Ethnicity, equity and public benefit: a critical evaluation of public umbilical cord blood banking in Australia. Bone Marrow Transplant 2007;40:729-34

23. Ballen KK, Wilson M, Wuu J, et al. Bigger is better: maternal and neonatal predictors of hematopoietic potential of umbilical cord blood units. Bone Marrow Transplant 2001; 27:7-14.

24. Romero FR, Xavier LR, Romero AW, et al. Heterogeneous methodology of racial/ethnic classification may be responsible for the different risk assessments for prostate cancer between Black and White men in Brazil. Int Braz J Urol 2015;41:360-6.

25. Al-Sweedan SA, Musalam L, Obeidat B. Factors predicting the hematopoietic stem cells content of the umbilical cord blood. Transfus Apher Sci 2013;48:247-52.

26. Solves P, Perales A, Moraga R, et al. Maternal, neonatal and collection factors influencing the haematopoietic content of cord blood units. Acta Haematol 2005;113:241-6.

27. George TJ, Sugrue MW, George SN, et al. Factors associated with parameters of engraftment potential of umbilical cord blood. Transfusion 2006;46:1803-12.

28. Bart T, Boo M, Balabanova S, et al. Impact of selection of cord blood units from the United States and Swiss registries on the cost of banking operations. Transfus Med Hemother 2013;40:14-20.

29. Clark P, Trickett A, Stark D, et al. Factors affecting microbial contamination rate of cord blood collected for transplantation. Transfusion 2012;52:1770-7.

30. Roh EY, Shin S, Kim BJ, et al. Roles of obstetricians in quality management of cord blood collection and informing potential donors: survey on obstetricians' understanding of cord blood in Korea. Transfusion 2014;54:3164-72. 